\title{
Structural Biochemistry
}

National Cancer Institute

\section{Source}

National Cancer Institute. Structural Biochemistry. NCI Thesaurus. Code C19047.

Studies concerned with the understanding of biological processes in terms of the structural properties of the macromolecules that participate in them. 